\title{
Charge Transfer in Magnetic Heterostructures from Atomic Resolution EELS
}

\author{
J. Aguiar, ${ }^{1,2}$ Q.M. Ramasse, ${ }^{3}$ M. Arredondo, ${ }^{4}$ F. Sandiumenge,${ }^{5}$ P. Abellan, ${ }^{5}$ N. Valanoor \\ ${ }^{4}$ and N.D. Browning ${ }^{1,2,6}$ \\ ${ }^{1}$ Condensed Matter and Materials Division, Lawrence Livermore National Laboratory, \\ Livermore, CA 37831-6064 \\ ${ }^{2}$ Dept. of Chem. Eng. and Mat. Sci., University of California Davis, Davis, CA 95616 \\ ${ }^{3}$ SuperSTEM Laboratory, STFC Daresbury, Keckwick Ln., Warrington, WA4 4AD, UK \\ ${ }^{4}$ School of Mat. Sci. and Eng., University of New South Wales, Sydney NSW, Australia \\ ${ }^{5}$ Institut de Ciència de Materials de Barcelona, ICMAB-CSIC Campus de la UAB 08193 \\ Bellaterra, Spain \\ ${ }^{6}$ Dept. of Molecular Cell Biology, University of California Davis, Davis, CA 95616
}

In the rapidly advancing field of spintronics, magnetic multilayered heterostructures and thin-film devices are being strongly considered to exploit magnetoelectric, magnetoelastic, and magnetoresistive effects. Candidate materials for this technology are manganese perovskites such as $\mathrm{La}_{1-x} \mathrm{Sr}_{\mathrm{x}} \mathrm{MnO}_{3}$ (LSMO) films grown on several substrates that showcase unique magnetic and magnetostrictive properties [1,2]. Potentially revolutionary functionalities are being manifested in manganite-based heterostructures, such as magnetotransport in LSMO/BaTiO and LSMO/STO multilayers coupled with magnetoelectric effects at interfaces $[3,4]$. The observed electrical and magnetic properties in thin films, multilayer devices, and nanostructures are largely determined by the interfacial structure and associated local chemical environment. By understanding how structural, magnetic, and transport properties are affected by interfacial structure, therefore can lead to elucidating the fundamental design principles for devices with improved performances. To address these materials design criteria a great deal of past experimental work has been already been performed to investigate interfacial effects on induced magneto effects on a variety of substrates [5,6]. In this study, AC STEM and electron energy loss spectroscopy (EELS) will be used in a comparative study to unravel the chemical interplay leading to charge transfer at interfaces between the perovskite structured LSMO and other similar and dissimilar structured substrates, including LSMO/BFO, LSMO/STO, LSMO/MgO and LSMO/PZT.

In an effort to unravel the fundamental underpinnings associated with charge transfer in each of these interfaces we initiated the study and have detected and coupled chemical inhomogeneties and charge fluctuations electronic structure intermixing with firstprinciples based theoretical calculations. To decouple the results we are focusing on the use of spectral fingerprints as an insight into the angular resolved DOS to resolve the metallic $d$-states that are largely responsible for observed electronic and magnetic effects in these materials. In particular, using the experimental oxygen $K$ and metallic $L$-edge EEL spectra as input we have conducted a series of varying DFT calculations using the fully linearized augmented plane wave (FP-LAPW) approach [7], as illustrated below in Figure 1 for LSMO, to determine the appropriate treatment of the electronic structure in these materials and understand the effects of the metallic $d$-states by varying different exchange and interaction potential values on the resolved spectra. 
Based on these results, we have converged on the set of parameters needed to treat and interpret the fundamental materials physics associated with anti/ferromagnetic interfaces and the effects of the metallic $d$ states based on a series of electronic structures calculations, respectively. The results of these calculations and the effects of structure coupled with the weighted correspondence with experiment will be further highlighted and presented at the conference.

\section{References:}

[1] S. Mercone et. al. , J. Supercond. 18, 1192005.

[2] S. Jin et al., Science 264, 413 (1994).

[3] I. Pallecchi et al., Phys. Rev. B 71, 014406 (2005).

[4] A.A. Sidorenko et al., Phys. Rev. B 73, 054406 (2006).

[5] Z. Fanget al., Phys. Rev. Lett. 84, 3169 (2000).

[6] Y.P. Lee et al., Phys. Rev. B 73, 224413 (2006).

[7] P. Blaha et al., Phys. Commun. 59, 399 (1990).

[8] K. Schwarz et al., Comput. Phys. Commun. 147, 71 (2002).

[9] The authors acknowledge support of the National Center for Electron Microscopy, Lawrence Berkeley Lab, which is supported by the U.S. Department of Energy under Contract \# DE-AC0205CH11231. This work was performed under the auspices of the U.S. Department of Energy by Lawrence Livermore National Laboratory under Contract DE-AC52-07NA27344. The authors would also like to acknowledge Jone Zabaleta for the growth of the LSMO nanodots on MgO substrates.

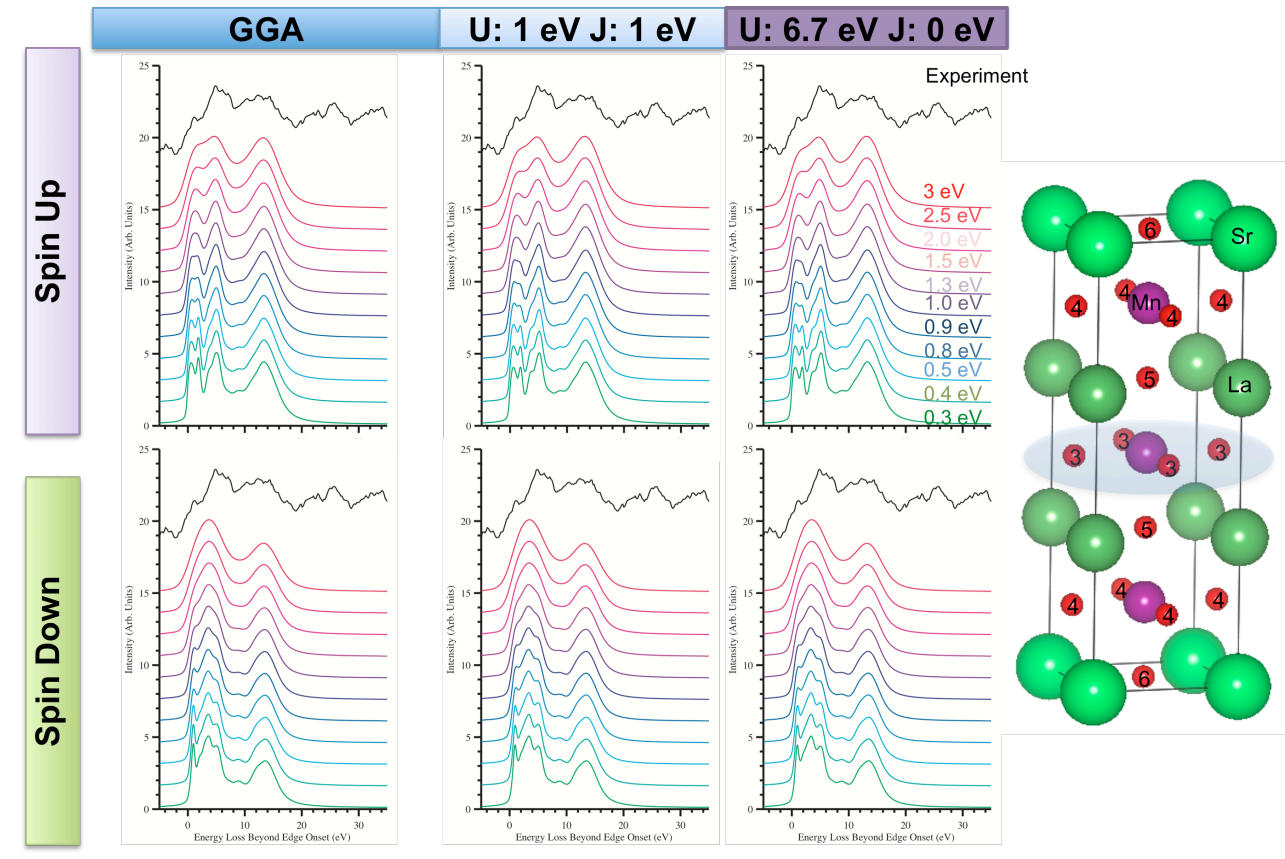

Figure 1: Comparing experiment and theory based on the $\mathrm{O} K$-edge for the pseudocubic approximation to LSMO considering broadening, spin, and the effective Hubbard U treatment. 